\title{
Percepción y valoración de servicios ambientales de las áreas protegidas de Puerto Deseado, Santa Cruz
}

\author{
Perception and valuation of environmental services in the protected areas of Puerto \\ Deseado, Santa Cruz
}

Patricia Alejandra GANDINI ${ }^{1,2}$,pagandini@yahoo.com.ar Ana MILLONES ${ }^{1}$, ana_millones@yahoo.com.ar

${ }^{1}$ Centro de Investigaciones de Puerto Deseado. Instituto de Ciencias del Ambiente, Sustentabilidad y Recursos Naturales, Unidad Académica Caleta Olivia. Universidad Nacional de la Patagonia Austral. Avenida Prefectura s/n (9050) Puerto Deseado Santa Cruz Argentina

${ }^{2}$ CONICET. Consejo Nacional de Investigaciones Científicas y técnicas.

Recibido: 26/11/2018. Aceptado: 03/04/2019

\section{RESUMEN}

La valoración económica de los recursos naturales ayuda a identificar opciones que permitan compatibilizar el desarrollo de una localidad y la conservación de sus ambientes naturales. Una de las metodologías que suele emplearse para estimar dicha valoración es la Disposición a Pagar (DAP). El objetivo de nuestro trabajo fue evaluar la DAP por los servicios ecosistémicos que brindan dos áreas protegidas de la localidad de Puerto Deseado. Para ello se utilizó el método de valoración de contingente, que a través de la realización de encuestas permite estimar la DAP de los individuos por la provisión o mejora de un bien ambiental. Para el armado de la encuesta se consideraron diferentes dimensiones: socioeconómicas; de preferencias de visita; estado de las áreas protegidas; problemas detectados; disponibilidad y modo de pago para conservar el status ambiental de las áreas protegidas. Se encuestó un total de 126 personas (74 residentes y 52 turistas). Para determinar cuál o cuáles variables consideradas pueden determinar la DAP se utilizaron análisis univariados y modelos de regresión logística. El modelo que mejor explicó la DAP incluyó como variables predictivas: lugar de residencia, si posee o no empleo y la percepción sobre el estado de las áreas protegidas. Según las encuestas, y en comparación a la respuesta de los turistas, la comunidad de Puerto Deseado mostró una mayor disposición a invertir en mantener o mejorar el estado de conservación de ambas áreas. Personas con empleo y con mayor salario mostraron una mayor DAP positiva. Los que están dispuestos a pagar demandan mayor control, vigilancia e infraestructura en las áreas protegidas. La fauna y paisaje local son los principales motivos de visitas, lo que señala la importancia de políticas de gestión tendientes a conservar el entorno lo más prístino posible. Se espera que los resultados obtenidos en este trabajo, sumados a futuros, sirvan para que el concepto de valoración de los servicios ecosistémicos pueda ser aplicado en una gestión más equitativa y efectiva de las áreas protegidas y sus recursos naturales.

Palabras clave: Áreas Protegidas; Servicios Ambientales; Valoración de Contingente. 


\begin{abstract}
The economic valuation of natural resources helps to identify options that make compatible conservation with development. One of the methodologies that is commonly used to this economic valuation is the willingness to pay (WTP). Our objective was to evaluate the WTP for the ecosystem services provided by two protected areas in Puerto Deseado. The contingent valuation method was used as an economic valuation tool. A survey was designed which included different dimensions: socioeconomic; visit preferences; current status of protected areas; detected problems; willingness and mode of payment to preserve or improve the environmental status of the protected areas. A total of 126 surveys (74 residents of Puerto Deseado and 52 tourists) were made. To test which variables can determine the WTP we employed univariate analyzes and logistic regression models. The model that best explained the WTP included as predictive variables: place of residence, have or not have a job, and the perception about the state of the protected areas. The community of Puerto Deseado showed a greater positive WTP to preserve or improve the environmental status of the protected areas. People with a job, and higher salaries, showed a higher positive DAP. Those people who were willing to pay, demanded a greater control, vigilance and better infrastructure in the protected areas. The local fauna and landscape are the main reasons for the visits, which indicates the importance of environmental policies tending to preserve the areas as pristine as possible. We are expected that our results, added to future ones, will help to apply the concept of economic valuation of ecosystem services for a more equitable and effective management of protected areas and their natural resources.
\end{abstract}

Key words: Protected Areas; Environmental Services; Contingent Value.

\title{
INTRODUCCIÓN
}

Las áreas protegidas, como los Parques Nacionales y las Reservas, son consideradas desde hace mucho tiempo como una herramienta clave para conservar la biodiversidad. Debido a que el crecimiento de la población humana está generando cada vez más impacto sobre los ecosistemas, ya sea amenazando su biodiversidad o modificando su dinámica natural de funcionamiento, cada día las áreas protegidas están cobrando mayor relevancia (Crutzen, 2002; Rockstrom et al., 2009). Sin embargo, dado el aumento de la necesidad por parte de los Estados de generar nuevas fuentes de trabajo, se está incrementando la intensidad de las actividades extractivas de recursos naturales, como por ejemplo la pesca y la minería. Esto plantea una dicotomía entre conservación y desarrollo, o entre preservación y progreso. Surge entonces un concepto estratégico denominado servicio ecosistémico. Nuevos paradigmas consideran a las áreas protegidas como parte del capital natural de los países por suministrar servicios ecosistémicos esenciales para el mantenimiento de la biodiversidad y que influyen en el bienestar de las poblaciones locales y de las sociedades en general. En los últimos cuarenta años se ha discutido extensamente el rol de la biodiversidad como un elemento que permita mejorar el bienestar social de las comunidades (Roe, 2008). Hoy en día se emplean técnicas de valoración económica que traducen esos servicios ecosistémicos en valores monetarios. Esta estrategia de valoración económica, además de ser una herramienta útil para la implementación de políticas que contribuyan a la conservación del ecosistema y del uso adecuado de sus recursos naturales, es una herramienta que puede ser utilizada a la hora de definir el modo en que puede ser solventada un área protegida. Una de las metodologías que suele emplearse para estimar el valor económico de un activo ambiental es la Disposición a Pagar (DAP) (Zappi y Cerda, 2011). 
El objetivo de nuestro trabajo fue evaluar la disposición a pagar por los servicios ecosistémicos que brindan las áreas protegidas de la localidad de Puerto Deseado. Como hipótesis planteamos que la disposición a pagar por servicios ambientales depende de factores socio-demográficos.

\section{Lugar de trabajo}

La ciudad de Puerto Deseado se encuentra en la provincia de Santa Cruz, sobre la margen norte de la Ría Deseado. Dicha localidad cuenta con dos áreas protegidas vecinas: la Reserva Provincial Ría Deseado (24.285ha, creada en 1977 y ampliada su categoría por ley en 2010) y el Parque Interjurisdiccional Marino Isla Pingüino (159.526 ha, creado en el 2010). El desarrollo de Puerto Deseado se basa en actividades relacionadas con la economía primaria, como la pesca y la ganadería, y últimamente también con las extractivas, como lo es la minería. Desde hace poco más de una década se viene desarrollando también, aún de manera incipiente, el turismo de naturaleza.

\section{MATERIALES Y MÉTODOS}

Para evaluar la disposición a pagar por los servicios ecosistémicos que brindan ambas áreas protegidas de la localidad de Puerto Deseado se utilizó el Método de Valoración Contingente, el cual consiste en realizar encuestas para estimar la máxima disposición a pagar de un individuo por la provisión o mejora de un bien (en este caso ambiental) que no se halla en el mercado o, alternativamente, la mínima disposición a ser compensado por la pérdida o disminución del disfrute del mismo bien (para más información sobre esta metodología consultar Zappi y Cerda, 2011).

Las variables y dimensiones consideradas para las encuestas se muestran en la Tabla 1.

Tabla 1. Dimensiones y variables incluidas en la encuesta realizada a miembros de la comunidad de Puerto Deseado y a turistas que visitaron la localidad.

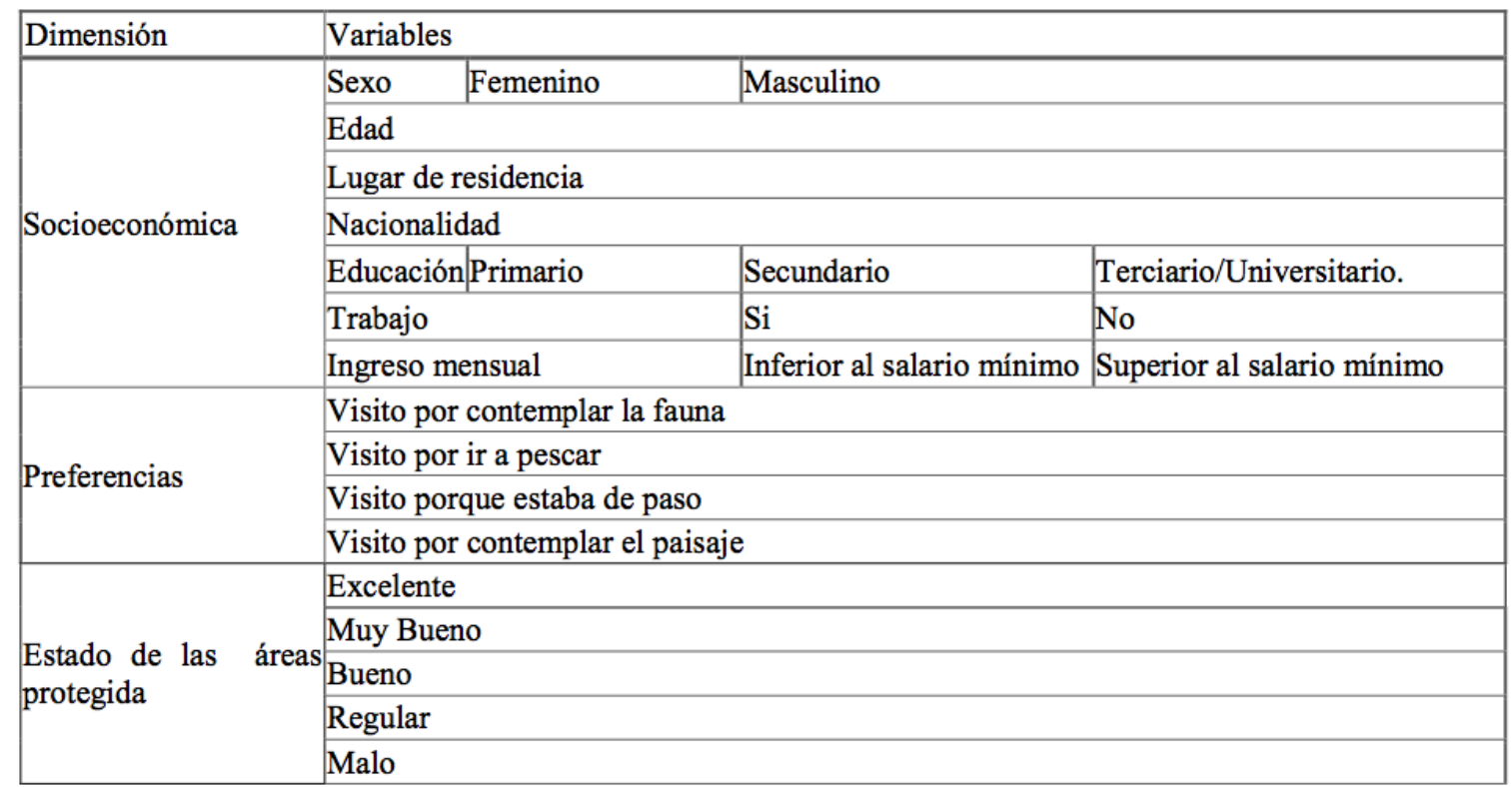




\begin{tabular}{|c|c|c|}
\hline \multirow{4}{*}{ Problemas detectados } & \multicolumn{2}{|l|}{ Falta de control } \\
\hline & \multicolumn{2}{|l|}{ Falta de Infraestructura } \\
\hline & \multicolumn{2}{|l|}{ Basura } \\
\hline & \multicolumn{2}{|l|}{ Destrucción de hábitat } \\
\hline \multirow{2}{*}{$\begin{array}{l}\text { Disponibilidad de pago por conservar el status ambiental } \\
\text { de la Reserva y el Parque o mejorarlo }\end{array}$} & \multicolumn{2}{|l|}{$(\mathrm{SI}-\mathrm{NO})$} \\
\hline & \begin{tabular}{ll|l} 
Modalidad & de & Bono \\
pago: & contribución
\end{tabular} & Obligatorio $\begin{array}{l}\text { No } \\
\text { contesta }\end{array}$ \\
\hline
\end{tabular}

Las encuestas fueron realizadas por un encuestador y de manera personal. Se trabajó con dos grupos poblacionales distintos: residentes de Puerto Deseado y turistas que visitaban la localidad. Los residentes de Puerto Deseado fueron encuestados cuando transitaban por la calle, mientras que para encuestar a turistas se recurrió a grupos de pasajeros de uno de los operadores náuticos de la localidad. Previo a la realización de las preguntas, a cada encuestado se le realizó una breve introducción de manera oral, explicando el sentido de la encuesta y se le aclaró que las respuestas quedarían de manera anónima. Una vez realizadas las encuestas, para determinar cuál o cuáles variables consideradas pueden determinar la DAP y así poder describir y predecir el comportamiento de la población, se utilizaron análisis univariados (test de Chi-cuadrado) y multivariados (modelos de regresión logística). Para estos últimos se consideró como variable respuesta la DAP (de manera binaria: "no pagaría" $=0$, "pagaría" $=1$ ) y como variables explicativas aquellas incluidas en la encuesta (ver Tabla $1)$.

\section{RESULTADOS}

Se le realizó la encuesta a un total de 126 personas: 74 residentes de Puerto Deseado y 52 turistas. El 66,6\% de los encuestados $(\mathrm{N}=84)$ estaba dispuesto a pagar (DAP $=1)$ para que el Parque Interjurisdiccional Marino Isla Pingüino y la Reserva Provincial Ría Deseado conserven el estatus ambiental o que mejoren. Se observó que la DAP no es independiente de las variables analizadas $\left(\chi^{2}=87,7 ; \mathrm{P}<0,0001\right)$. El modelo de regresión logística que mejor explicó la DAP incluyó como variables predictivas lugar de residencia (si pertenece o no a la comunidad de Puerto Deseado), trabajo (si posee o no empleo) y la percepción sobre el estado de las áreas protegidas $\left(\mathrm{AUC}_{\text {modelo }}=0,885, \chi^{2}=87,75 ; \mathrm{P}=0,0001\right)$. Analizando por separado cada una de las variables incluidas en el modelo de regresión logística se pudo observar que la mayoría de los turistas entrevistados se manifestaron negativamente en cuanto a la DAP, mientras que una alta proporción de miembros residentes de la comunidad de Puerto Deseado lo hicieron de manera positiva (Fig. 1). 


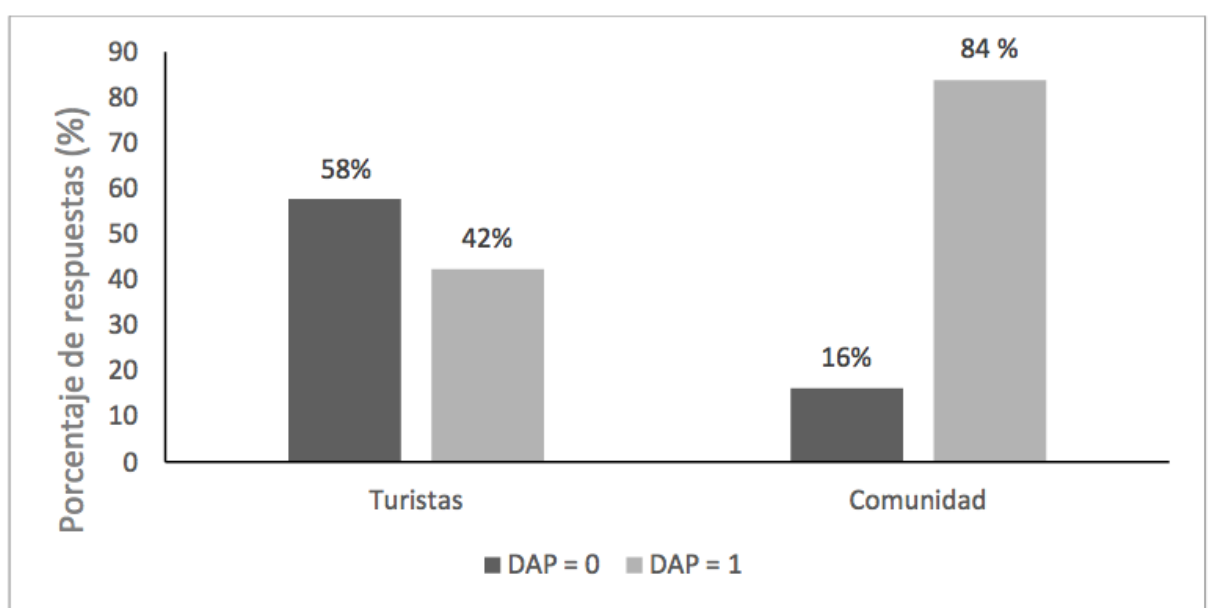

Figura 1. Porcentaje de respuestas positivas y negativas respecto a la disposición a pagar por servicios ambientales según si los encuestados eran residentes de Puerto Deseado (comunidad) o turistas.

En cuanto a la percepción sobre el estado de las áreas protegidas es claro que la mayoría, sin importar si es de la comunidad residente o turista, cuando perciben que el estado de conservación es malo o regular tienen una respuesta positiva en cuanto a la DAP, al igual que cuando el estado es excelente o muy bueno. Para los que perciben que el estado de conservación es tan solo bueno esta variable de percepción sobre el estado no es buena para discriminar por si sola la DAP (Fig. 2).

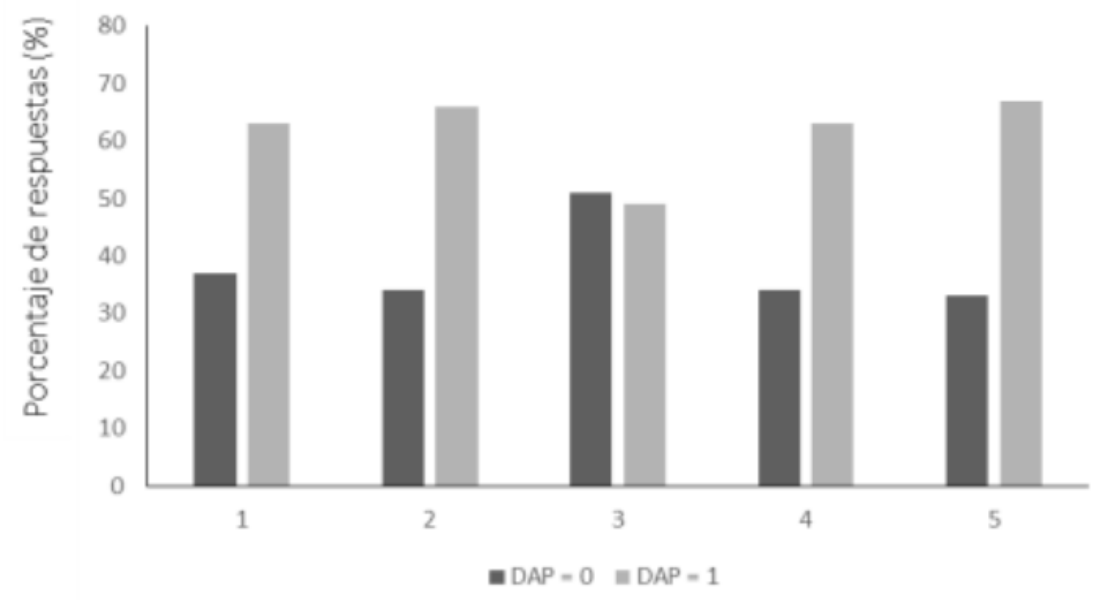

Figura 2. Porcentajes de respuestas positivas y negativas en cuanto a la disposición a pagar o no por servicios ambientales según la percepción del estado de las áreas protegidas: 1. Excelente; 2. Muy Bueno; 3. Bueno; 4. Regular; 5. Malo

Cuando se analizó la variable trabajo se observó un mayor porcentaje de respuestas positivas $(\mathrm{DAP}=1)$ por aquellos que trabajan (Fig. 3 ). 


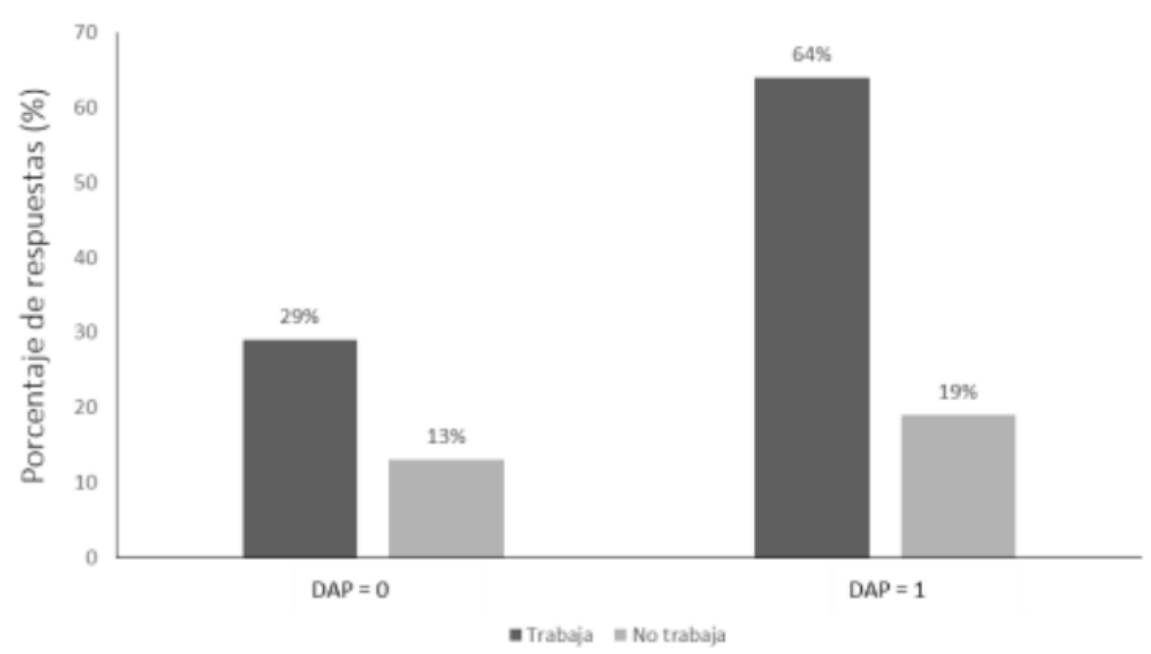

Figura 3. Porcentajes de respuestas positivas y negativas en cuanto a la disposición a pagar o no por servicios ambientales según situación laboral al momento de la encuesta: trabaja / no trabaja.

Cuando se analizó la DAP en relación al ingreso mensual para residentes argentinos (se excluyó del análisis las respuestas de los turistas extranjeros, ya que los ingresos por ser en dólares o euros no son comparables con el de los residentes) se observó un mayor porcentaje de $\mathrm{DAP}=1$ por parte de aquellas personas que perciben un salario superior al mínimo. El resto de las variables sociodemográficas analizadas (sexo, educación y edad) no explicaron la DAP.

$\mathrm{Al}$ analizar las respuestas de los encuestados en cuanto al estado de las áreas protegidas y los problemas detectados en las mismas (un encuestado puede haber señalado más de una categoría o ninguna), se observó que un $65 \%$ de las personas $(\mathrm{N}=82)$ detectaron a la basura como un problema en las áreas protegidas. Esta detección pareciera ser independiente de la DAP (el $48 \%$ mostró una disposición negativa a pagar, mientras que el $52 \%$ restante mostró una disposición positiva al pago). En cuanto a la falta de infraestructura, solo el $20 \%$ de los encuestados lo detectó como un problema de las áreas protegidas. Esta detección pareciera también no ser tan diferente entre los que estarían dispuestos a pagar de los que no lo estarían ( $44 \%$ vs $56 \%$, respectivamente). En cuanto a la falta de control solo un $25 \%$ de los encuestados la detectó como problema, pero aquí sí fue claro que de aquellos que la detectaron la mayoría (80\%) estarían dispuestos a pagar (Tabla 2).

Tabla 2. Porcentaje de encuestados que detectaron los distintos problemas en las áreas protegidas y porcentaje de los que estaban o no dispuestos a pagar.

\begin{tabular}{|l|l|l|l|}
\hline & $\begin{array}{l}\text { Porcentaje de } \\
\text { encuestados que lo } \\
\text { detectaron }\end{array}$ & $\begin{array}{l}\text { No dispuestos a pagar } \\
(\mathrm{DAP}=0)\end{array}$ & $\begin{array}{l}\text { Dispuesto a pagar } \\
(\mathrm{DAP}=1)\end{array}$ \\
\hline Basura & $65 \%(\mathrm{~N}=82)$ & $48 \%(\mathrm{~N}=39)$ & $52 \%(\mathrm{~N}=43)$ \\
\hline $\begin{array}{l}\text { Falta de } \\
\text { Infraestructura }\end{array}$ & $20 \%(\mathrm{~N}=25)$ & $56 \%(\mathrm{~N}=14)$ & $44 \%(\mathrm{~N}=11)$ \\
\hline Falta de control & $25 \%(\mathrm{~N}=25)$ & $20 \%(\mathrm{~N}=5)$ & $80 \%(\mathrm{~N}=20)$ \\
\hline $\begin{array}{l}\text { Destrucción de } \\
\text { hábitat }\end{array}$ & $0 \%$ & 0 & 0 \\
\hline
\end{tabular}


En cuanto a la forma de pago se obtuvo un total de $\mathrm{N}=54$ respuestas correspondiendo a un $43 \%$ del total de encuestados $(\mathrm{N}=126)$. Cuarenta y seis personas $(85 \%)$ respondieron que la misma debería ser un monto obligatorio, siendo entonces ésta la modalidad de pago más aceptada, seguida por la opción de un bono contribución, con el $15 \%$ de los encuestados $(\mathrm{N}=$ 8). Un total de 72 entrevistados no respondieron a esta pregunta.

En cuando a las preferencias o motivo de la visita a las áreas protegidas, solo 90 encuestados respondieron este punto. De estos, un $32 \%(\mathrm{~N}=40)$ señalaron que su visita se debió a las bellezas escénicas de las áreas, un $22 \%(\mathrm{~N}=28)$ respondieron que visitaron las áreas protegidas para ir a pescar, el $16 \%(\mathrm{~N}=20)$ las visitaron para contemplar la fauna del lugar, y solo dos personas respondieron que visitaron las áreas protegidas porque estaban de paso.

\section{CONCLUSIONES Y DISCUSIONES}

Las áreas protegidas son el soporte económico de muchas comunidades, crean trabajo y contribuyen al orgullo local de las comunidades vecinas. Para lograr ello es necesario complementar e integrar el manejo de los entornos naturales con las actividades productivas que se desarrollan (Berghöfer y Förster, 2010). En la Patagonia costera se llevan adelante diferentes actividades productivas primarias, algunas de ellas por explotación directa de los recursos, como por ejemplo la obtención de productos alimenticios (servicios ambientales de aprovisionamiento como la pesca y la cosecha de algas) y otras por explotación indirecta, como por ejemplo el turismo o recreación (servicios ambientales culturales) que junto a los servicios ambientales de soporte como el reciclado de nutrientes) no son valorados hasta que los ecosistemas son degradados. Para poder llevar adelante el desarrollo de actividades económicas y que vayan de la mano con la conservación es clave considerar la biodiversidad y su ambiente como parte del capital natural. Es entonces cuando surge la necesidad de valorar económicamente los servicios ecosistémicos que presta un ambiente natural, y una manera de hacerlo es a través del método de valoración de contingente. Nuestro trabajo destaca cuestiones clave a tener en cuenta a la hora de poner en valor los servicios ecosistémicos que brindan las dos áreas protegidas vecinas a la localidad de Puerto Deseado. Según las encuestas, la comunidad de Puerto Deseado, en su mayoría, está dispuesta a invertir para que las áreas de su entorno continúen con el mismo estado de conservación en el que actualmente se encuentran, e inclusive invertir para su mejora. Por el contrario, una proporción alta de los visitantes no estaba dispuesta a pagar, lo cual es llamativo dado que las visita a esta localidad están generalmente motivada por el atractivo de su entorno natural y/o cultural. Seguramente la inversión que demanda, tanto a turistas nacionales como extranjeros, el trasladarse a Patagonia, especialmente Puerto Deseado alejado $300 \mathrm{~km}$ de la terminal aérea más cercana, es alta por lo que cualquier "ahorro" en términos de su viaje es apreciado.

A través de las encuestas quedó claro que los que están dispuestos a pagar demandan mayor control y vigilancia, como también más infraestructura para un mayor disfrute de las áreas protegidas. Parece ser clave también, que a la hora de decidir invertir en conservación, el hecho de tener o no trabajo afecta directamente en dicha decisión. Otro punto destacable de los resultados de las encuestas es que en la mayoría de los casos se consideró que la forma de pago debería ser obligatorio.

El paisaje y la fauna local fueron los principales motivos por los cuales los encuestados decidieron visitar las áreas protegidas. Es por ello que en las políticas de gestión en ambas 
áreas debe considerarse conservar el entorno lo más prístino posible y tratar de controlar los problemas clave, como lo es por ejemplo la gestión de los residuos urbanos.

En Argentina, los aportes para mantener las áreas protegidas los hacen los Estados, ya sea el nacional, provincial o municipal. Estos suelen poseer magros presupuestos anuales y normalmente no tienen relación con el bienestar que las áreas protegidas brindan a través de sus servicios ecosistémicos (Figueroa et al., 2009). En nuestro país no suelen existir medidas de valoración que incorporen los servicios ambientales y su contribución como insumos a las actividades productivas de explotación directa o indirecta. A través de los resultados preliminares obtenidos en este trabajo, sumados a los que obtendremos en futuros estudios que desarrollaremos en la provincia de Santa Cruz, y en el resto del país, esperamos que este concepto de valoración de los servicios ecosistémicos pueda ser aplicado en una gestión más equitativa y efectiva de nuestras áreas protegidas y sus recursos naturales.

\section{AGRADECIMIENTOS}

A los alumnos de la Maestría en Gestión y Manejo de Recursos Naturales - UNPA - que participaron del "Curso Manejo y Diseño de Reservas" y realizaron encuestas y a Roxana Goronas, de Darwin Expediciones, quien colaboró con la realización de las encuestas a los turistas.

\section{BIBLIOGRAFÍA}

BERGHÖFER, A. y FÖRSTER, J. (2010). Ecosystem Services and Protected areas. Chapter 7. En: The Economics of Ecosystems and Biodiversity (Heidi Wittmer Eds), 126-140.

CRUTZEN, P.J., (2002). The effects of industrial and agricultural practices on atmospheric chemistry and climate during the Anthropocene. Journal of Environmental Science and Health, 37(4), 423-424, http://dx.doi.org/10.1081/ESE-120003224

FIGUEROA E., REYES P. Y ROJAS V. (2009). Pago por Servicios Ambientales en Áreas Protegidas en América Latina. Fortalecimiento del Manejo Sostenible de los Recursos Naturales en las Áreas Protegidas de América Latina . FAO. https://doi.org/10.18242/anpscripta.2016.02.02.01.0005

ROCKSTRÖM, J., STEFFEN, W., NOONE, K., PERSSON, A., CHAPIN, F.S., LAMBIN, E.F., LENTON, et al. (2009). A safe operating space for humanity. Nature, 46(1), 472475, http://dx.doi.org/10.1038/461472a

ROE, D. (2008) The origins and evolution of the conservation-poverty debate: a review of key literature, events and policy processes. Oryx, 42(4), 491-503, https://doi.org/10.1017/S0030605308002032

ZAPPI, M. \& C. CERDA. 2011. ¿Qué motiva a las personas a conservar? El rol del biocentrismo en la valoración económica de la conservación. Revista Ambiente Forestal, 6(10), 14-21. 
Esta obra está bajo una Licencia Creative Commons Atribución-NoComercial-SinDerivar 4.0 Internacional. 